\title{
Pressure Fluctuations on the Impeller Blades of a Centrifugal Turbomachine: a Comparative Analysis Between Air and Water Tests
}

\author{
A. Talha \\ Laboratoire de Mécanique de Lille (L.M.L. U.R.A CNRS 1441), I.U.T "A", GMP, Villeneuve d'Ascq Cedex, F-59653, France \\ J.P. Barrand and G. Caignaert \\ Laboratoire de Mécanique de Lille (L.M.L. U.R.A CNRS 1441), E.N.S.A.M, Lille Cedex, F-59046, France
}

(Received 13 December 2000; revised 20 November 2001; accepted 28 November 2001)

\begin{abstract}
In this paper a comparison between pressure fluctuations measurements in the time domain is proposed. These measurements were carried out on two centrifugal turbomachines, a fan with air and a pump with water. In order to gather a better understanding of their internal acoustics, time records of the pressure fluctuations were made on the blades in the outlet of the impellers and near the volute tongue. These showed that the flow is very unsteady at the impeller outlet and in the tongue region. An analysis of the pressure fluctuations and their propagation showed that the main noise sources are caused by the blade-tongue interactions and depend on the fluid type. In the case of the pump, the hydraulic nature of the noise source is dominant. Using a one-dimensional propagation model, it is possible to explain some differences in the behaviour of the turbomachines which is related in particular to the different sound speeds.
\end{abstract}

\section{NOMENCLATURE}

$P A, P W \quad$ - pressure transducer on the pressure side of the blade for air and water respectively

$S A, S W$ - pressure transducer on the suction side of the blade for air and water respectively

$T A, T W \quad$ - pressure transducer near the volute tongue for air and water respectively

$D_{1}, D_{2} \quad$ - inlet and outlet diameters of the impeller

$D_{3}, D_{4} \quad$ - inlet and outlet diameters of the diffuser

$N \quad$ - pump rotational speed (rpm)

$Q, Q_{n} \quad$ - volume flow rate, design flow rate $\left(\mathrm{m}^{3} / \mathrm{s}\right)$

$H_{i}, H_{n} \quad$ - internal head, nominal head (m)

e $\quad$ - impeller outlet width ( $\mathrm{mm})$

$Z \quad$ - number of blades

$p \quad$ - pressure $(\mathrm{Pa})$

$M_{1}, M_{2} \ldots$ - peak pressure fluctuation, mechanical effect

$H_{1}, H_{2} \ldots$ - peak pressure fluctuation, hydraulic effect

$t \quad$ - time (s)

$T \quad$ - rotational period (s)

$x \quad-\operatorname{abscissa}(\mathrm{m})$

$P_{e} \quad$ - impeller perimeter (m)

$u \quad$ - impeller peripheral tip speed $(\mathrm{m} / \mathrm{s})$

$C_{u} \quad$ - absolute circumferential flow velocity

$f_{r} \quad$ - rotation frequency $(\mathrm{Hz})$

$f_{z} \quad$ - blade passing frequency $(\mathrm{Hz})$

$\rho \quad$ - fluid density $\left(\mathrm{kg} / \mathrm{m}^{3}\right)$

$\omega \quad$ - frequency $(\mathrm{rad} / \mathrm{s})$

$\omega_{s} \quad$ - dimensionless specific speed

$\psi \quad-$ head coefficient

$\delta \quad-$ flow coefficient

a $\quad$ - speed of sound $(\mathrm{m} / \mathrm{s})$

$v \quad$ - kinematic viscosity $\left(\mathrm{m}^{2} / \mathrm{s}\right)$

$g \quad$ - acceleration of gravity $\left(\mathrm{m} / \mathrm{s}^{2}\right)$

$\varphi \quad$ - angular position (degrees)
$R_{e} \quad-$ Reynolds number

$M_{a} \quad$ - Mach number

\section{INTRODUCTION}

The primary sources of noise in centrifugal turbomachines are associated with the interactions of the non-uniform outflow from the impeller with the volute tongue or the leading edges of the diffuser blades as has already demonstrated by Tyler \& Sofrin (1961) in the case of axial flow in compressors and by Copley (1962) in the case of radial flow in pumps.

As shown by Chu et al. (1993) and Abramian and Howard (1994), pressure fluctuations measured on the casing are difficult to interpret and are linked to the structure of the flow, owing to the simultaneous occurrence of several unsteady flow phenomena. Further studies of Sinha and Katz (2000) confirmed this fact and the effects such as the impact of blade wakes on the tongue, blockage of flow passages, vortex development and tongue vibrations. The best way to reduce the noise generation is to increase the gap between the impeller and the casing up to a limit of $20 \%$, after which the machine performance is reduced (Dong et al. 1997).

However, if a detailed analysis is essential for a good understanding of the noise production mechanisms, it is also important to gather other information as well. This includes a knowledge of the wave generation inside the machine, and how the pressure fluctuations are generated and merged in organised waves inside it to produce the hydraulic noise in the inlet and outlet pipes. The local pressure fluctuations do not depend uniquely on the local features of the flow. But they depend also on perturbations initiated at other places inside or outside the machine. These perturbations propagate along different paths in the casing (diffuser and volute) and in the flow passages of the impeller. 